\title{
CONTROLE DE Rhipicephalus sanguineus (LATREILLE, 1806) (ACARI: IXODIDAE) NO CANIL DA ESCOLA DE VETERINÁRIA DA UFMG, BELO HORIZONTE, MINAS GERAIS, BRASIL
}

\author{
GUSTAVO F. PAZ1; ROMÁRIO C. LEITE²; PAULO R. DE OLIVEIRA ${ }^{2}$
}

\begin{abstract}
PAZ, G.F.; LEITE, R.C.; OLIVEIRA, P.R. DE. [Control of Rhipicephalus sanguineus (Latreille, 1806) (Acari: Ixodidae) in the kennel of the UFMG Veterinary School, Belo Horizonte, Minas Gerais, Brazil]. Controle de Rhipicephalus sanguineus (Latreille, 1806) (Acari: Ixodidae) no canil da Escola de Veterinária da UFMG, Belo Horizonte, Minas Gerais, Brasil. Revista Brasileira de Parasitologia Veterinária, v. 17, n. 1, p.41-44, 2008. Escola de Veterinária, Universidade Federal de Minas Gerais, Av. Antonio Carlos, 6627, Pampulha, Belo Horizonte, MG 31270-901. E-mail: rcleite@vet.ufmg.br

The present work was aimed at proposing a control measure for Rhipicephalus sanguineus in a naturally infected kennel of $72 \mathrm{~m}^{2}$ of the UFMG Veterinary School with 25 dogs of different breeds. A sensitivity test to acaricide products was applied in a sample of $R$. sanguineus. Out of the acaricide products tested: $12.5 \%$ Amitraz (product 1); $2 \%$ Alfamethrin and 60\% Dichlorvos association (product 2); $5 \%$ Deltamethrin (product 3 ) and the association between $77.6 \%$ Trichlorfon, 1.0\% Coumaphos and 1.0\% Cyfluthrin (product 4); only 1 and 4 products showed $100 \%$ efficacy. Amitraz (12.5\%) was used for controlling $R$. sanguineus in the surrounding of the kennel, on which four treatments every seven days were carried out in the facilities. For parasitic $R$. sanguineus, a single topical treatment was used: Flumetrin $1.0 \%$ pour - on on all the 25 dogs. This control measure significantly reduced the population of $R$. sanguineus within the kennel. A slight infestation was observed in the seventh month after treatment. A new application of $12.5 \%$ Amitraz was done in the kennel as well as a new topical treatment with Flumetrin $1.0 \%$ pour - on on the dogs. The control procedure proposed in the present study was an emergency measure and its effectiveness was checked through environmental surveys throughout a one-year period.
\end{abstract}

KEY WORDS: Rhipicephalus sanguineus, control, acaricides.

\section{RESUMO}

O presente estudo teve como proposta controlar uma infestação natural de Rhipicephalus sanguineus em um canil pertencente à Escola de Veterinária da UFMG, com uma área de $72 \mathrm{~m}^{2}$ e 25 cães de diversas raças. Um teste de sensibilidade a produtos acaricidas foi aplicado em uma amostra de carrapatos capturados no canil. Dos produtos testados: Amitraz 12,5\% (Produto 1); uma associação de Alfametrina 2\% e Dichlorvós 60\% (produto 2); Deltametrina 5\% (produto 3) e uma associação de Trichlorfon 77,6\%, Coumaphós 1\% e Cyfluthrin 1\%

\footnotetext{
${ }^{1}$ Centro de Pesquisa René Rachou, Fundação Oswaldo Cruz, Av. Augusto de Lima, 1715, sala 219, Barro Preto, Belo Horizonte, MG 30190-002. Email: gustavopaz@cpqrr.fiocruz.br

${ }^{2}$ Escola de Veterinária, Universidade Federal de Minas Gerais, Av. Antonio Carlos, 6627, Pampulha, Belo Horizonte, MG 31270-901. E-mail: rcleite@vet.ufmg.br
}

(produto 4); somente os produtos 1 e 4 apresentaram 100\% de eficácia. O Amitraz 12,5\% foi utilizado no controle dos estádios de $R$. sanguineus presentes no ambiente do canil, com um esquema de 4 aplicações a cada 7 dias de intervalo. Para os estádios parasitários, um único tratamento com Flumetrina $1.0 \%$ pour-on foi empregado em todos os animais. A inspeção e o monitoramento do ambiente e dos animais foram realizados durante um período de um ano. Estas medidas de controle reduziram significativamente a população de carrapatos dentro do canil. O canil não apresentou infestação pelo carrapato nos seis meses seguintes aos tratamentos. Uma infestação leve foi observada no $7^{\circ}$ mês pós-tratamento, quando foi realizada uma nova aplicação de Amitraz 12,5\% no ambiente do canil e um novo tratamento com Flumetrina 1.0\% pour-on nos animais, com resultados satisfatórios.

PALAVRAS-CHAVE: Rhipicephalus sanguineus, controle, acaricidas. 


\section{INTRODUÇÃO}

O carrapato da espécie Rhipicephalus sanguineus, além de ser um dos principais problemas parasitários enfrentados por proprietários de canis, vem se destacando cada vez mais no ambiente domiciliar e peridomiciliar do homem que convive com o principal hospedeiro urbano deste ectoparasito, o cão doméstico Canis familiaris.

A espécie $R$. sanguineus tem sido encontrada em alta prevalência em cães urbanos, sendo descrita na literatura como uma das principais espécies de parasitos destes animais domésticos (LINARDI; NAGEM, 1973; LABRUNA; PEREIRA, 2001; SZABÓ et al., 2001; GONZÁLEZ et al., 2004; SOARES et al., 2006). Está comprovadamente envolvida na transmissão de agentes patogênicos, destacando - se entre estes: Babesia canis e Eherlichia canis (GOTHE et al.,1989; SMITH et al., 1976).

Com freqüência, torna-se numa importante praga urbana que começa a requerer atenção dos organismos de saúde publica, sendo ainda, motivo de constante preocupação entre os profissionais veterinários em seus locais de atendimento.

Infestações de $R$. sanguineus em humanos foram notificadas nos seguintes municípios do Brasil: Goiana, GO, Recife, PE, Pedreira, SP e Cachoeira do Sul, RS (GUGLIELMONE et al., 2006). Assim, por se desenvolver em ambientes sinantrópicos de várias cidades do país, onde ocorre em alta densidade e prevalência, este carrapato poderá vir a causar aumento na incidência de Erliquiose, Babesiose e Febre Maculosa, como antropozoonoses emergentes (FERNANDES et al., 2001).

Ainda que estudos da biologia de $R$. sanguineus sejam freqüentes, a literatura que trata de seu controle em condições naturais de infestação é escassa, destacando-se os trabalhos que avaliam a eficácia de colares impregnados com acaricidas (VAN DEN BOS; CURTIS, 2002; ESTRADA-PEÑA; RÈME, 2005) e produtos de uso tópico (JERNIGAN et al., 2000; ENDRIS et al., 2002; YOUNG et al., 2003; DRYDEN et al., 2006) em cães infestados experimentalmente com este carrapato. Na literatura pesquisada, apenas os trabalhos de Labruna e Pereira (2001) e Labruna (2004) abordam aspectos da eco epidemiologia deste carrapato no Brasil, que são importantes para o direcionamento das medidas de controle de $R$. sanguineus em ambientes naturalmente infestados.

Este trabalho teve como objetivo propor medidas de controle de $R$. sanguineus em um canil naturalmente infestado.

\section{MATERIAL E MÉTODOS}

O estudo foi realizado no canil do Hospital Veterinário da Escola de Veterinária da Universidade Federal de Minas Gerais no período de abril de 2001 a maio de 2002, motivado pela reclamação dos responsáveis pelo prédio de uma infestação em elevados níveis, persistente e incontrolada a despeito do uso contínuo de carrapaticidas.

O canil, com uma área total de $72 \mathrm{~m}^{2}$ e com uma lotação permanente de 25 cães de raças variadas divididos em 11 baias, possuía, no inicio do experimento, uma elevada infestação natural de carrapatos. Esta infestação foi observada em uma primeira inspeção no ambiente e nos animais. Inicialmente, foram coletadas do ambiente fêmeas ingurgitadas de carrapatos desprendidas naturalmente do hospedeiro. A coleta foi realizada durante três dias consecutivos às 7:00 h da manhã. Após a identificação, segundo Aragão e Fonseca (1961), as fêmeas ingurgitadas foram limpas com pincel de cerdas naturais $\mathrm{n}^{\circ} 2$, lavadas em água destilada, secas em papel filtro e pesadas em balança analítica.

Sessenta (60) fêmeas ingurgitadas foram selecionadas e submetidas ao teste de sensibilidade a produtos acaricidas de acordo com Drumond et al. (1973), com o objetivo de selecionar o produto químico a ser utilizado no controle dos carrapatos. Foram utilizadas as seguintes bases acaricidas: Amitraz 12,5\% (Produto 1); uma associação de Alfametrina 2\% e Dichlorvós 60\% (produto 2); Deltametrina 5\% (produto 3) e uma associação de Trichlorfon 77,6\%, Coumaphós 1\% e Cyfluthrin 1\% (produto 4). Dois grupos de 10 teleóginas cada foram mantidos como controle.

Em função da eficácia observada e preço de mercado dos produtos, foi escolhido o produto comercial com a base química Amitraz 12,5\%, que foi preparado em um barril plástico (diluição comercial de $2 \mathrm{ml} /$ /itro de água) e utilizado no controle químico das formas não parasitárias de $R$. sanguineus presentes no ambiente do canil.

Foram realizadas quatro aplicações do produto com sete dias de intervalo, sendo a primeira aplicação realizada na primeira semana do mês de maio de 2001. As aplicações foram realizadas de 7:00 às 8:00 h da manhã utilizando-se uma bomba pulverizadora elétrica (marca WAP), de alta pressão e baixo consumo. A quantidade de aplicações foi baseada no acompanhamento da carga parasitária de carrapatos no ambiente do canil e nos animais, de modo que, na última aplicação, não deveria ser observado carrapatos no ambiente e nos animais. O método de avaliação para a presença de carrapatos foi qualitativo e não quantitativo, tendo sido observado a presença ou não de carrapatos no ambiente e nos animais durante o período de estudo. A inspeção semanal se estendeu por um período de um ano após a primeira aplicação do produto.

O produto foi aplicado em toda a área do canil como pisos, grades, paredes e tetos. Foram preparados, para cada aplicação, 200 litros de solução carrapaticida, sendo gastos aproximadamente 2,8 litros da solução carrapaticida por $\mathrm{m}^{2}$. Para o combate das formas parasitárias utilizou-se em cada animal (25 cães) um único tratamento tópico com Flumetrina 1\% pour - on. O critério para a escolha do produto pour - on foi o melhor preço de mercado somado à sua boa eficácia, conforme relatos dos médicos veterinários do Hospital Veterinário da UFMG.

Do início ao fim do experimento, a cada substituição de um animal alojado, o cão recém chegado era submetido ao mesmo tratamento pour - on previamente determinado, com o objetivo de impedir novas infestações ambientais, sem impedir a rotina de utilização da instalação. 


\section{RESULTADOS E DISCUSSÃO}

Todas as teleóginas coletadas no canil, utilizadas para o teste de sensibilidade a produtos acaricidas, foram da espécie $R$. sanguineus. Este fato evidencia uma alta freqüência deste carrapato em uma população de cães domésticos provenientes de diversas áreas da região metropolitana de Belo Horizonte, MG.

Dentre os produtos acaricidas utilizados no teste de sensibilidade, apenas os produtos 1 (Amitraz 12,5\%) e 4 (associação Trichlorfon 77,6\% , Coumaphós 1\% e Cyfluthrin 1\%) tiveram $100 \%$ de eficácia. Todas as teleóginas tratadas com estes produtos não realizaram postura, quando comparadas às do grupo controle, onde $100 \%$ das teleóginas a realizaram. Já os produtos 2 (associação Alfametrina 2\% e Dichlorvós 60\%) e 3 (Deltametrina 5\%) tiveram 51,8\% e 0\% de eficácia respectivamente. A baixa eficácia dos produtos 2 e 3 pode estar relacionada com a resistência da população de carrapatos do canil a estes produtos, uma vez que existe o histórico do uso indiscriminado de produtos à base de Piretróide no ambiente do canil.

Os tratamentos realizados no ambiente com o produto 1 na diluição comercial de $2 \mathrm{ml} /$ litro de água, associado ao tratamento tópico dos animais com Flumetrina pour on, controlou de maneira eficiente a população de carrapato no canil, fato este, observado através das inspeções semanais realizadas no local.

O canil permaneceu livre de $R$. sanguineus por um período de seis meses. Uma leve infestação ambiental foi observada no sétimo mês após o início do tratamento, provavelmente decorrente de alguma falha no controle preventivo dos carrapatos à entrada no canil. Um fato importante a se considerar é o tempo esperado de ação terapêutica do produto pour - on, podendo assim ter ocorrido algum desprendimento de estádios parasitários no ambiente do canil devido à ação não imediata deste produto. Tendo em vista este fato uma nova aplicação tática com o produto 1 foi realizada no canil, bem como o tratamento tópico de todos os animais com o produto pour on utilizado anteriormente. Nenhuma infestação foi observada posteriormente até o término do período de inspeção, realizada na última semana do mês de maio de 2002.

O esquema de tratamento proposto para o controle químico de $R$. sanguineus reduziu de maneira expressiva a carga de infestação por este carrapato no canil, durante o período de um ano de avaliação. É importante salientar que a inspeção do ambiente, assim como dos animais, deve ser feita sistematicamente durante e após as medidas de controle; com o objetivo de monitorar possíveis reinfestações de $R$. sanguineus, determinando assim a freqüência de tratamentos químicos a serem realizados.

O controle das infestações por $R$. sanguineus em cães se faz principalmente com o uso de drogas carrapaticidas, seja no meio ambiente e/ou nos cães (LABRUNA; PEREIRA, 2001). A droga de escolha para o tratamento no ambiente, onde se encontram 95\% da população de carrapatos, tem sido à base de Piretróide, por apresentar maior poder residual, su- gerindo-se de três a quatro aplicações com intervalos de 14 dias (LABRUNA, 2004). Neste trabalho a droga de escolha foi o produto à base de Amitraz devido à sua melhor eficácia apresentada no teste de sensibilidade a produtos acaricidas proposto por Drumond et al. (1973). O intervalo de 7 dias entre as aplicações foi devido ao menor período residual desta base química quanto comparada às bases do grupo dos Piretróides. Assim, sugere - se que a droga de escolha para o tratamento ambiental seja de eficácia comprovada por um teste de sensibilidade a produtos acaricidas, o intervalo entre as aplicações deve ser estipulado com base na duração média do período residual da droga de escolha e a quantidade de aplicações de acordo com o acompanhamento da carga de infestação de carrapatos no local, através das inspeções periódicas no ambiente e nos animais.

Outras medidas preventivas para evitar a entrada de carrapatos no canil podem ser utilizadas como: a inspeção cuidadosa em todo o pêlo do animal, principalmente nos espaços interdigitais, cabeça, pescoço, dorso e orelhas, que são frequentemente infestados como relatado por Labruna (2004); a manutenção dos cães em uma baia telada por um período mínimo de 15 dias juntamente com a aplicação de um produto pour - on e a manutenção e conservação da estrutura do canil para impedir o surgimento de possíveis frestas e buracos, tendo em vista que todas as fases de desenvolvimento em vida livre do carrapato (mudas, posturas e incubação dos ovos) se passam em frestas ou buracos presentes nas paredes ou teto destes locais onde o cão vive, conforme relatos de Labruna e Pereira (2001).

O esquema de controle químico proposto neste trabalho foi tático e emergencial com o uso de produtos acaricidas no ambiente e nos animais. Maiores estudos sobre a duração do ciclo deste carrapato em condições naturais e a sua dinâmica populacional no Brasil devem ser realizados para que medidas de controle estratégico possam ser adotadas futuramente.

\section{REFERÊNCIAS BIBLIOGRÁFICAS}

ARAGÃO, H.B.; FONSECA, F. Notas de Ixodologia VIII. Lista e chave para os representantes da fauna ixodológica brasileira. Memórias do Instituto Oswaldo Cruz, v. 59, n. 1, p. 115-29, 1961.

DRUMMOND, R.O.; ERNEST, S.E.; TREVINO, J.L.; GLADNEY, W.J.; GRAHAM, O.H. Boophilus annulatus and B. microplus: laboratory tests of insecticides. Journal of Economic Entomology, v. 66, n. 1, p. 130-133, 1973.

DRYDEN, M.W.; PAYNE, P.A.; SMITH, V.; HOSTETLER, J. Evaluation of an imidacloprid $(8.8 \% \mathrm{w} / \mathrm{w})$ - permethrin $(44.0 \% \mathrm{w} / \mathrm{w})$ topical spot-on and a fipronil $(9.8 \% \mathrm{w} / \mathrm{w})-$ (S)-methoprene (8.8\% w/w) topical spot-on to repel, prevent attachment, and kill adult Rhipicephalus sanguineus and Dermacentor variabilis ticks on dogs. Veterinary Therapeutics : research in applied veterinary medicine, v. 7, n. 3, p. 187-198, 2006.

ENDRIS, R.G.; HAIR, J.A.; KATZ, T.L.; ZOBRE, E.; PENNINGTON, R.G.; MEYER, J.A. Efficacy of three dose 
volumes of topically applied 65\% permethrin against Ctenocephalides felis and Rhipicephalus sanguineus on dogs weighing $30 \mathrm{~kg}$ or more. Veterinary Therapeutics : research in applied veterinary medicine, v. 3, n. 4, p. 435 440, 2002.

ESTRADA-PEÑA, A.; RÈME, C. Efficacy of a collar impregnated with amitraz and pyriproxyfen for prevention of experimental tick infestations by Rhipicephalus sanguineus, Ixodes ricinus, and Ixodes scapularis in dogs. Journal of the American Veterinary Medical Association, v. 226, n. 2, p. 221 - 224, 2005.

FERNANDES, F.F.; FREITAS, E.P.; SILVA, J.R.; SILVA, O.R.; SILVA, I.G. Efeitos toxicológicos e ineficiência in vitro de deltametrina sobre larvas de Rhipicephalus sanguineus, de Goiânia, Goiás, Brasil. Revista da Sociedade Brasileira de Medicina Tropical, v. 34, n.2, p. 159165, 2001.

GONZÁLEZ, A.; CASTRO DDEL, C.; GONZÁLEZ, S. Ectoparasitic species from Canis familiaris (Linné) in Buenos Aires province, Argentina. Veterinary Parasitology, v. 120, n. 1-2, p.123-129, 2004.

GOTHE, R.; WEGEROT, S.; WALDEN, R.; WALDEN, A. Epidemiology of Babesia canis and Babesia gibsoni infections in dogs in Germany. Kieintierpraxis, v. 34, n. 7, p.309-320, 1989.

GUGLIELMONE, A.A.; BEATI, L.; BARROS-BATTESTI, D.M.; LABRUNA, M.B.; NAVA, S.; VENZAL, J.M.; MANGOLD, A.J.; SZABO, M.P.; MARTINS, J.R.; GONZÁLEZ-ACUÑA, D.; ESTRADA-PEÑA, A. Ticks (Ixodidae) on humans in South America. Experimental and Applied Acarology, v. 40, n. 2, p. 83-100, 2006.

JERNIGAN, A.D.; MCTIER, T.L.; CHIEFFO, C.; THOMAS, C.A.; KRAUTMANN, M.J.; HAIR, J.A.; YOUNG, D.R.; WANG, C.; ROWAN, T.G. Efficacy of selamectin against experimentally induced tick (Rhipicephalus sanguineus and
Dermacentor variabilis) infestations on dogs. Veterinary Parasitology, v. 91, n. 3, p.359-375, 2000.

LABRUNA, M.B.; PEREIRA, M.C. Carrapato em Cães no Brasil. Clínica Veterinária, v. 30, n. 1, p. 24-32, 2001.

LABRUNA, M. B. Biologica-ecologia de Rhipicephalus sanguineus (Acari: Ixodidae). Revista Brasileira de Parasitologia Veterinária, v.13, Supl., p.123-124, 2004.

LINARDI, P.M.; NAGEM, R.L. Pulicídeos e outros ectoparasitos de cães de Belo Horizonte e municípios vizinhos. Revista Brasileira de Biologia, v. 33, n. 4, p. 529538, 1973.

SMITH, R.D.; SELLS, D.M.; STEPHENSON, E.H.; RISTIC, M.; HUXOLL, D.L. Development of Ehrlichia canis, causative agent of canine ehrlichiosis, in the tick Rhipicephalus sanguineus and its differentiation from a symbiotic rickettsia. American Journal of Veterinary Research, v. 37, n. 2, p.119-126, 1976.

SOARES, A.O.; SOUZA, A.D.; FELICIANO, E.A.; RODRIGUES, A.F.; D'AGOSTO, M.; DAEMON, E. Evaluation of ectoparasites and hemoparasites in dogs kept in apartments and houses with yards in the city of Juiz de Fora, Minas Gerais, Brazil. Revista Brasleira de Parasitologia Veterinária, v. 15, n. 1, p.13-6, 2006.

SZABÓ, M.P.; CUNHA, T.M; PINTER, A.; VICENTINI, F. Ticks (Acari: Ixodidae) associated with domestic dogs in Franca region, São Paulo, Brazil. Experimental and Applied Acarology, v. 25, n. 10, p.909-916, 2001.

VAN DEN BOS, R.H.; CURTIS, R.J. The use of a $4 \%(\mathrm{w} / \mathrm{w})$ deltamethrin collar (Scalibor Protector Band) in the extended control of ticks on dogs. Experimental and Applied Acarology, v. 28, n. 1-4, p.297-303, 2002.

YOUNG, D.R.; ARTHER, R.G.; DAVIS, W.L. Evaluation of K9 Advantix vs. Frontline Plus topical treatments to repel brown dog ticks (Rhipicephalus sanguineus) on dogs. Parasitology Research, v. 90, n. 3, p. 116-118, 2003.

Recebido em 13 de agosto de 2007.

Aceito para publicação em 12 de dezembro de 2007. 